\title{
Long-term benefits of nevirapine-containing regimens: multicenter study with 506 patients, followed-up a median of 9 years
}

D. Podzamczer, ${ }^{1}$, J.M. Tiraboschi ${ }^{1}$, J. Mallolas ${ }^{2}$, J. Curto ${ }^{1}$, M.A. Cárdenes ${ }^{3}$, E. Casas ${ }^{4}$, A. Castro ${ }^{5}$, S. Echevarría ${ }^{6}$, M. Leal ${ }^{7}$, J.C. Lopez Bernaldo de Quirós ${ }^{8}$, S. Moreno ${ }^{9}$, T. Puig $^{10}$, E. Ribera ${ }^{11}$, C. Villalonga ${ }^{12}$, J.L. Gómez-Sirvent ${ }^{13}$, J.A. García-Henarejos ${ }^{14}$, J. Lopez-Aldeguer $^{15}$, P. Barrufet ${ }^{16}$, L. Force ${ }^{16}$, I. Santos ${ }^{17}$ and J. Sanz ${ }^{17}$

${ }^{1}$ Hospital Universitari de Bellvitge, L'Hospitalet de Llobregat, Barcelona, ${ }^{2}$ Hospital Clinic de Barcelona, ${ }^{3}$ Hospital Dr. Negrín, Las Palmas, ${ }^{4}$ Hospital Alcalá de Henares, Madrid, ${ }^{5}$ Hospital Universitario de La Coruña, ${ }^{6}$ Hospital de Valdecilla, Santander, ${ }^{7}$ Hospital Virgen del Rocío, Sevilla, ${ }^{8}$ Hospital Gregorio Marañón, Madrid, ${ }^{9}$ Hospital Ramón y Cajal, Madrid, ${ }_{10}$ Hospital Arnau de Vilanova, Lleida, ${ }^{11}$ Hospital Vall d'Hebron, Barcelona, ${ }^{12}$ Hospital Son Dureta, Palma de Mallorca, ${ }^{13}$ Hospital Universitario de Canarias, Santa Cruz de Tenerife, ${ }^{14}$ Hospital Santa $M^{a}$ del Rosell, Murcia, ${ }^{15}$ Hospital La Fe, Valencia, ${ }^{16}$ Hospital de Mataró, Barcelona, and ${ }^{17}$ Hospital La Princesa, Madrid, Spain

\begin{abstract}
Objective: To evaluate long-term outcomes in patients maintaining a nevirapine (NVP)-based regimen.

Methods: Retrospective, multicenter, cohort study including patients currently receiving an NVP regimen that had been started at least 5 years previously. Demographic, clinical, and analytical variables were recorded.

Results: Median follow-up was 8.9 (5.7-11.3) years. Baseline characteristics: $74 \%$ men, 47 years old, 36\% drug users, $40 \%$ AIDS, $40 \% \mathrm{HCV}+, 51.4 \%$ detectable HIV-1 viral load, CD4 count $395(4-1,421) / \mu \mathrm{L}, 19 \% \mathrm{CD} 4<200 / \mu \mathrm{L}, 27 \%$ ALT grade 1-2, 36\% AST grade 1-2. Thirty percent ART-naive, 83\% received NVP associated with 2 nucleoside analogues during the study period, and $17 \%$ a protease inhibitor.

A significant improvement was observed in general health status markers, including hemoglobin, platelets, and albumin, regardless of $\mathrm{HCV}$ coinfection. CD4 cell gain was +218 and $+322 / \mu \mathrm{L}$ after 6 and 9 years, respectively $(+321$ and +391 in naive patients). Triglycerides significantly decreased in pretreated patients, whereas the percentage of patients with HDLc $<1.03 \mathrm{mmol} / \mathrm{L}$ and LDL-c $>3.37 \mathrm{mmol} / \mathrm{L}$ significantly decreased in a subsample with available values. A significant decrease in transaminases, alkaline phosphatase, and Fib4 score was observed, mainly in HCV+ and ARV-naive patients.

Conclusions: In patients who tolerate NVP therapy, (even those with HCV coinfection), long term benefits may be significant in terms of a progressive improvement in general health status markers and CD4 response, a favorable lipid profile, and good liver tolerability.
\end{abstract}

Keywords: Nevirapine, antiretroviral therapy, long term benefits, tolerability, liver outcome, NVP, NNRTI, CD4, naive patients, regimens. 


\section{INTRODUCTION}

Combined antiretroviral therapy (cART) is the standard of care for HIV-infected patients. Among antiretroviral (ARV) regimens, a nucleoside backbone associated with a non-nucleoside reverse transcriptase inhibitor (NNRTI) is considered a first-line option in ART-naïve patients [1]. The NNRTI nevirapine (NVP) is one of the "oldest" currently available ARV drugs, first commercialized in 1997. Despite the development of many new ARV compounds since then, NVP is still considered a first option in European Guidelines [1] and an acceptable option in US guidelines [2], and continues to be prescribed in a considerable number of patients. One of the main advantages of NVP regimens is their lipid profile $[3,4]$, whereas hypersensitivity and liver toxicity are considered limitations [5]

Most clinical trials evaluating ARV regimens have a follow-up of one or two years. Longer experiences come from observational cohort studies or routine clinical practice. Although cART has been prescribed for about 15 years, there is little long-term published information regarding ARV regimens [6, 7].

HIV infection has become a chronic disease and patients need treatment for many years; hence, it is important to know the long-term tolerability of ARV regimens. The objective of this study was to assess the general health status, liver function, lipid changes, and CD4 counts of patients receiving NVP for at least five years. To our knowledge, this study contains the longest clinical follow-up data related to the use of NVP-containing regimens.

\section{METHODS}

This is a retrospective, observational, multicenter cohort study, including adult HIV-infected patients currently receiving a NVP-containing regimen that had been started more than 5 years previously. Patients initiating a nevirapine-containing regimen between January $1^{\text {st }} 1998$ and June $30^{\text {th }} 2003$ and continuing with nevirapine up to the end of July 2008, were included in the study. In centres where the number of patients still on nevirapine was greater than 30, we selected a random sample of 30 patients in each of them. Randomization was done centrally by computergenerated random numbers. The final sample size obtained (506 patients) was considered large enough to estimate the pre-defined end points.

The primary end points were liver, lipid, and CD4 outcomes. Baseline demographic information was documented, and the following data were recorded for yearly analysis: CD4 count, viral load, blood cells, and ALT, AST, alkaline phosphatase, GGT, glucose, creatinine, total cholesterol, triglycerides, HDL-c, and LDL-c levels. Data assessing liver fibrosis, such as FibroScan and liver biopsy, were recorded when available, and the Fib-4 test was determined using the formula: (age x AST) /(Platelets x square root of $A L T)[8]$.

\section{Statistical Analysis}

Results for continuous variables are presented as the median of the absolute values or as the median percentage change from baseline and the range (minimum and maximum). Results for categorical variables are presented as frequencies and percentages. Between-group comparisons of continuous variables were performed with the independent $t$-test for those with a normal distribution and the Mann Whitney $U$ test for those with a non-normal distribution. Comparisons between follow-up and baseline results of continuous variables were carried out with the $t$-test for repeated measures in those with a normal distribution and Wilcoxon's signed-rank test in those with a non-normal distribution. A two-sided significance level of 0.05 was used in all comparisons. Analyses were performed with PASW Statistics, version 18.0.0 (SPSS, Inc., 2009, Chicago, IL, www.spss.com). 


\section{RESULTS}

\section{Baseline Characteristics}

5521 patients initiated a nevirapine-containing regimen in the period 1998-2003 in the participating hospitals, and 1394 of them (25.2\%) remained taking nevirapine at the study enrollment (2008). After randomization, 506 patients were included in the study.

Baseline characteristics are shown in Table 1. Patients had been followed-up for a median of 8.9 (5.711.3) years: $270(53.3 \%)$ had been followed-up for 9 years and $506(100 \%)$ for 6 years. More than $95 \%$ of patients were white, native Spaniards: $26 \%$ were women, $36 \%$ drug users, $40 \%$ had AIDS, and $44 \%$ had HCV $(40.1 \%)$ or HBV (4\%) coinfection. Among coinfected patients, 61 (27.6\%) received therapy for chronic $\mathrm{HCV} / \mathrm{HBV}$, and 37 (16.7\%) presented a sustained virologic response. Median CD4 count was 395 (4- 1421 cells/ $\mu \mathrm{L})$, and HIV-1 viral load $(\mathrm{VL})$ was $<40(<40-831,250)$ copies/mL; $18.8 \%$ of patients had a CD4 count $<200$ cells $/ \mu \mathrm{L}$ and $54.5 \%$ had an undetectable HIV-1 VL. Thirty percent of patients were ART-naïve when initiating NVP and $69.9 \%$ were ART-experienced. Of the $51.4 \%(n=253)$ of patients initiating nevirapine with a detectable viral load at baseline, 102 were pretreated patients with previous virologic failure or discontinuation of prior therapies due to toxicities $(20.2 \%$ of the total). The current regimens most frequently used were: tenofovir/emtricitabine/ NVP in 31.2\%, abacavir/lamivudine/NVP in $23.7 \%$, and zidovudine/lamivudine/NVP in $22.3 \%$. Protease inhibitors (PI) -mainly indinavir and nelfinavir- were associated in $17.4 \%$ (all except 4 patients had been pretreated) whereas nucleoside analogues alone were combined with NVP in $83.6 \%$. At the end of follow-up, only $2.9 \%$ of patients maintained the PI (mainly lopinavir/ritonavir).

Among the total, $27.6 \%$ of patients presented ALT levels above the normal range (26.6\% grade $1-2$ and $1 \%$ grade 3 ), and $37.7 \%$ had AST above normal range (36.1\% and $1.6 \%$, respectively). 
Table 1. Baseline Characteristics and Laboratory Parameters

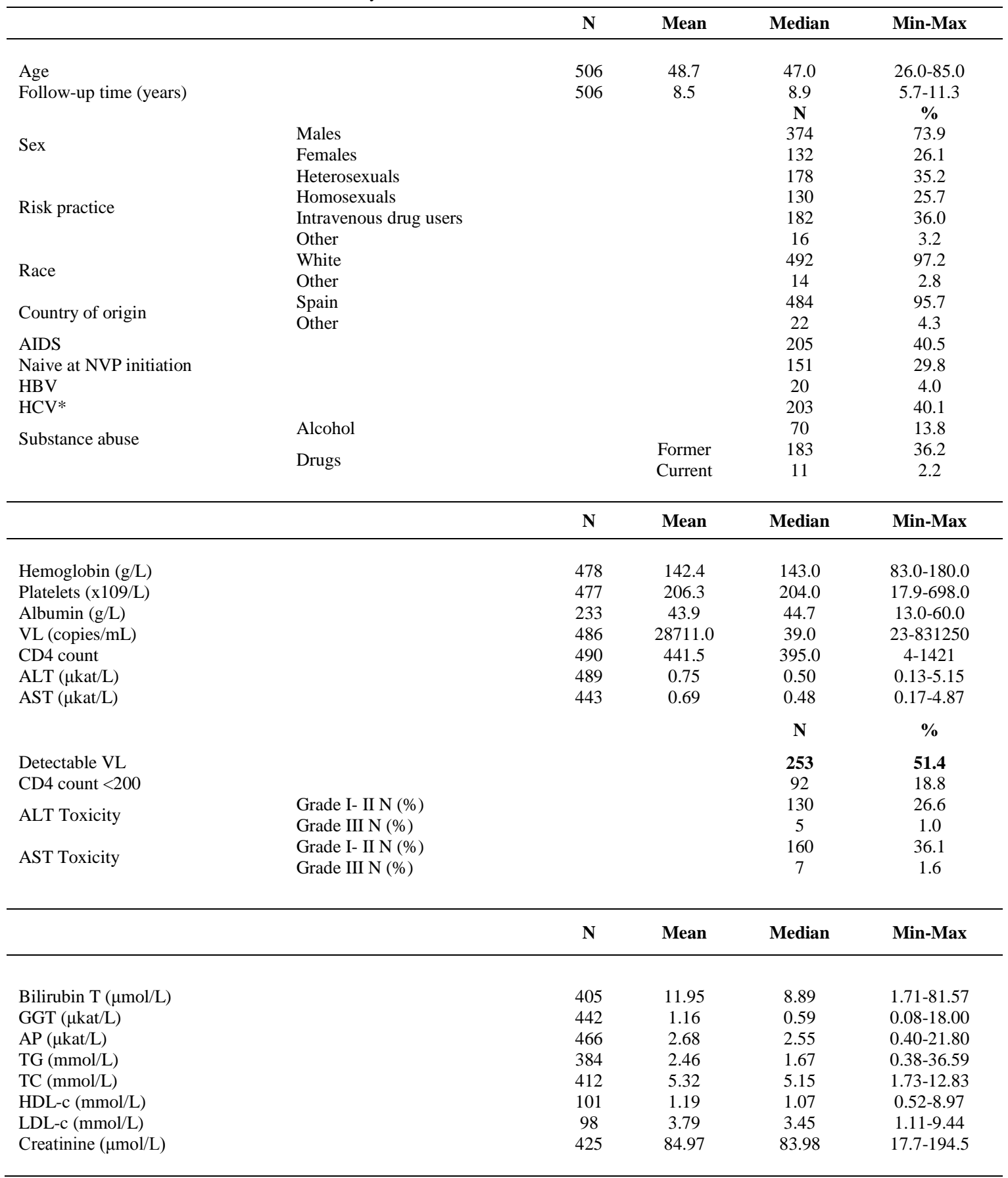

203 patients had HIV/HCV coinfection and a liver biopsy or FibroScan was performed in $83(49 \%)$; 47 (56\%) of them had significant fibrosis (Metavir Score $>2$ or FibroScan $>7 \mathrm{kPa}$ ).

AP, alkaline phosphatase; ALT, alanine transaminase; AST, aspartate transaminase; GGT, gamma-glutamyltransferase; T, total; TC, total cholesterol; TG, triglycerides; VL, viral load.

Grade I-II ALT: > ULN - 5 x ULN, Grade III-IV ALT: > 5.0 x ULN.

Grade I-II AST: > ULN - 5 x ULN, Grade III-IV AST: $>5.0$ x ULN.

ULN, upper limit of normal. 


\section{Outcomes}

\section{General Health Status}

Changes in hemoglobin levels, albumin levels, and platelet counts (Fig. 1): Hemoglobin levels significantly increased after 6 and 9 years of follow-up $(5 \mathrm{~g} / \mathrm{L}(3.34 \%), \mathrm{p}<0.001$; and $7 \mathrm{~g} / \mathrm{L}(4.76 \%)$, $\mathrm{p}<0.001)$ regardless of $\mathrm{HCV}$ coinfection $(\mathrm{HCV}+\mathrm{p}<0.001$ at 6 and 9 years, HCV $-\mathrm{p}<0.001$ at 6 and 9 years), although the increase was significantly greater in naïve patients at 6 years $(6 \mathrm{~g} / \mathrm{L} v s 4 \mathrm{~g} / \mathrm{L}, \mathrm{p}=0.014)$. Platelet counts were significantly higher in pretreated patients compared to naïve patients $(214,000 \mathrm{vs}$ $190,000 / \mu \mathrm{L}, \mathrm{p}<0.001)$. However, platelet increase was significantly greater after 6 and 9 years in ARTnaïve patients $(39,000$ [19.6\%] and 34,000 [17.6\%]) versus pretreated patients $(3,000$ [1.5\%] and 12,000 [7.1\%]) (both time points, p<0.001) (Fig. 2). Platelet count significantly increased in both $\mathrm{HCV}+$ and $\mathrm{HCV}$ - patients, with no differences between these groups. Similarly, albumin levels significantly increased regardless of $\mathrm{HCV}$ coinfection. Changes in general health status parameters were similar, regardless of concomitant PI use (data not shown).

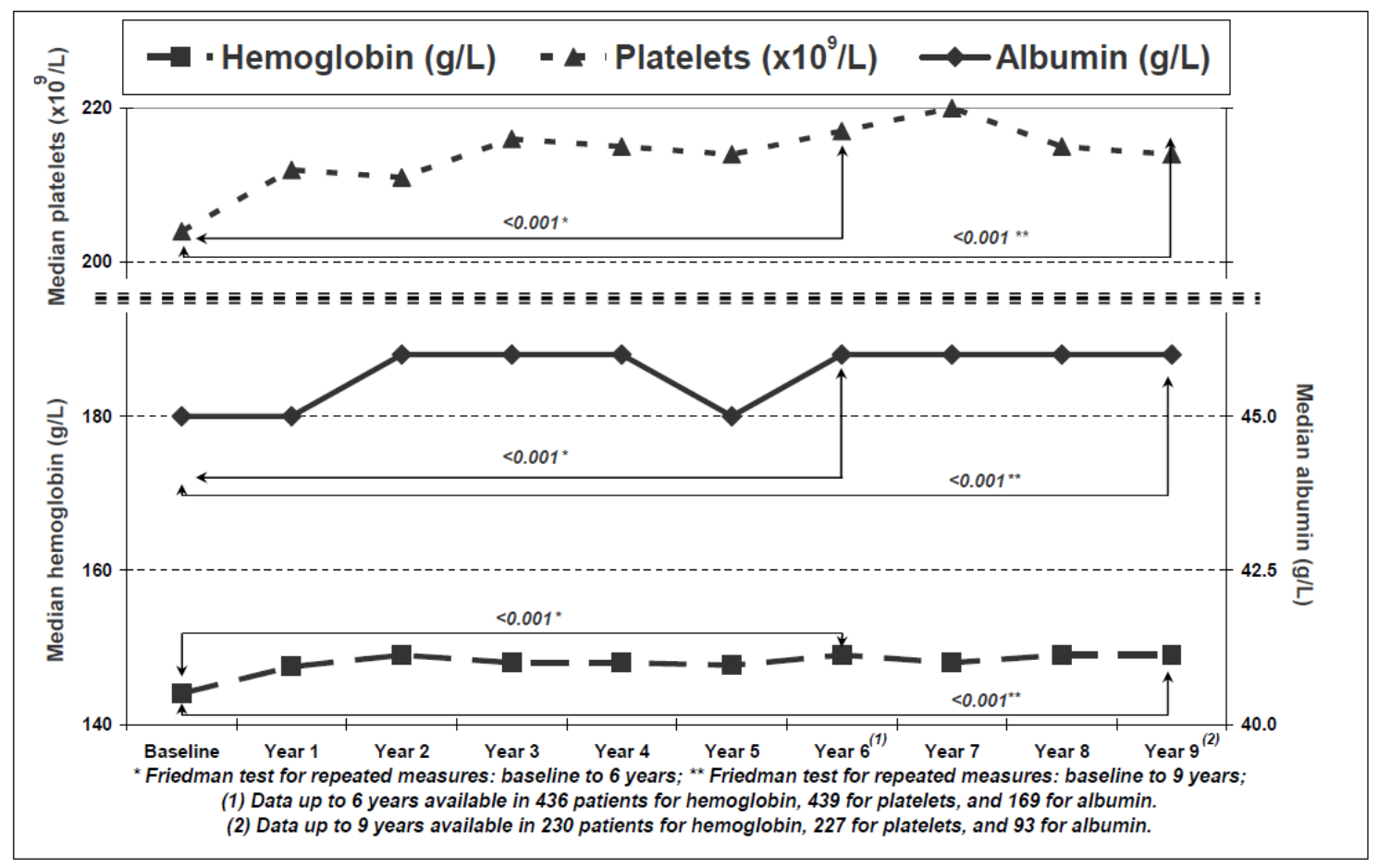

Fig. (1). General health status markers. 


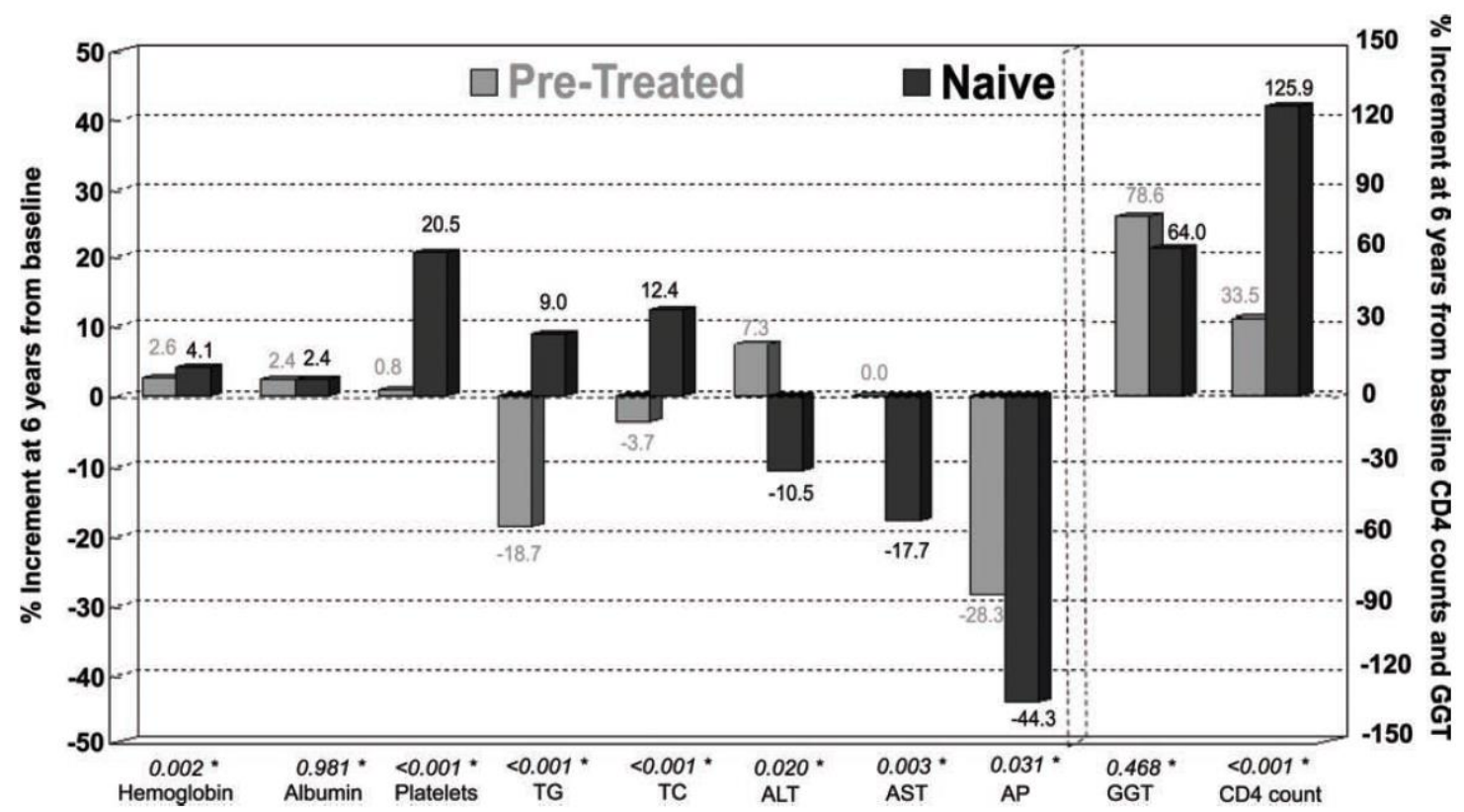

Fig. (2). Percentage change in laboratory parameters in naïve versus pretreated patients.

\section{CD4 Count and Viral Load}

In the overall cohort, CD4 count significantly increased at 6 and 9 years: +210 cells $/ \mu \mathrm{L}$ and +296 cells $/ \mu \mathrm{L}$, respectively (both, $\mathrm{p}<0.001$ ). In patients treated with 2 nucleoside analogues $+\mathrm{NVP}$, results were very similar: +218 cells $/ \mu \mathrm{L}$ and +322 cells $/ \mu \mathrm{L}$. The increase was greater in patients who did not have $\mathrm{HCV}$ coinfection $(+257 v s+176$ cells $/ \mu \mathrm{L}$ at 6 years, $\mathrm{p}=0.007)$ (Fig. 3$)$, and results were similar in those who were not receiving a PI. ART-naïve patients had lower baseline CD4 counts than pretreated patients $(\mathrm{p}<0.001)$ but no differences were observed after 6 and 9 years of follow-up because of a greater CD4 gain in naïve patients: $+321 v s+168$ cells $/ \mu \mathrm{L}(\mathrm{p}<0.001)$ at 6 years and $+391 v s+250$ cells $/ \mu \mathrm{L}$ $(\mathrm{p}<0.001)$ at 9 years (Fig. 2). While $51.4 \%$ of patients had detectable plasma HIV-1 viral load at baseline, viral load was undetectable in $96.7 \%$ patients $(\mathrm{n}=487)$ after 6 years and in $98.1 \%(\mathrm{n}=261)$ after 9 years.

\section{Lipid Changes}

In the total cohort, $25.7 \%$ of patients received lipidlowering drugs during the study period, including $22.7 \%$ of those not taking a PI and $39.8 \%$ of those taking a PI $(\mathrm{p}=0.004)$.

Triglycerides (TG) decreased by $8 \%$ at 6 years $(\mathrm{p}=0.009)$, but showed a nonsignificant increase of $3.6 \%$ at 9 years. With regard to $\mathrm{HCV}$ coinfection, TG decreased to a significantly greater extent in $\mathrm{HCV}+$ patients than in HCVpatients $(-17.6 \%$ vs $-1.2 \%$; $\mathrm{p}=0.024)$. TG levels were higher in pretreated patients $(\mathrm{p}<0.001)$, a significant decrease occurred only in pretreated patients $(\mathrm{p}<0.001)$, and the decrease $(-16 \%$, and $-18.6 \%$ in patients not taking a PI) significantly differed from the levels in naïve patients $(+9.3 \%$, $\mathrm{p}<0.001$ ) (Fig. 2). 
Total cholesterol increased at 9 years only in the subgroup of HCV-patients $(+6.4 \%, \mathrm{p}=0.006)$ and in naïve patients $(+11.5 \%, \mathrm{p}<0.001)$, with similar results in patients who were not receiving a PI.

HDL-c data, collected in a subsample of 46 patients, showed an increase at 6 years (overall $+19.7 \%$, $\mathrm{p}<0.001)$ in both naïve and pretreated patients regardless of HCV status. No significant changes in LDL-c were observed in 40 patients with available follow-up data.

At 6 years, the percentage of patients with HDL-c $<1.03 \mathrm{mmol} / \mathrm{L}$ decreased from $33.7 \%$ to $17.5 \%$ $(\mathrm{p}=0.011)$, and the percentage with LDL-c $>3.37 \mathrm{mmol} / \mathrm{L}$ decreased from $49.4 \%$ to $26.6 \%(\mathrm{p}=0.004)$. Results were similar in patients who were not taking a PI.

\section{Liver Status Changes}

Overall, there were no differences from baseline in ALT values at 6 and 9 years. Nonetheless, although HCV+ patients had significantly higher ALT levels at baseline $(0.75 \mu \mathrm{kat} / \mathrm{L} v s 0.40 \mu \mathrm{kat} / \mathrm{L}$, $\mathrm{p}<0.001)$, transaminase decreases were greater in this group at 6 years: $-3.6 \% v s+6.7 \%(\mathrm{p}=0.047)$ and $12.5 \%$ v $+5.9 \%(\mathrm{p}=0.026)$ in patients not receiving a PI. ALT decrease was greater in naïve patients: $10.5 \%$ vs $+7.3 \%(\mathrm{p}=0.020)$ at 6 years and $-11.0 \% v s+8.5 \%(\mathrm{p}=0.039)$ at 9 years (Fig. 2). A similar trend was observed for AST changes (Fig. 3).

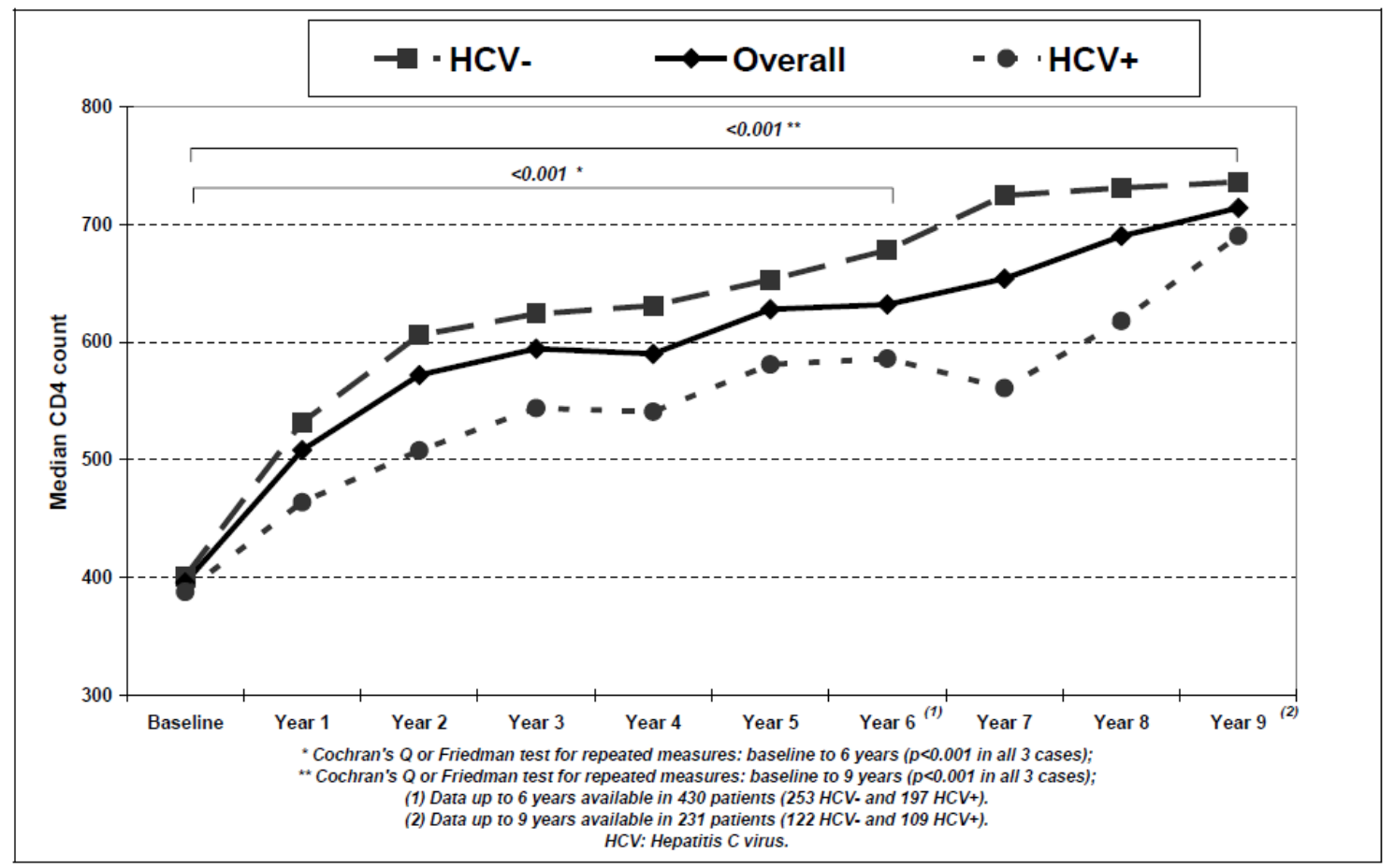

Fig. (3). CD4 counts versus HCV positive or negative status. 
As was expected, GGT increased by $78.1 \%$ and $117.3 \%$ at 6 and 9 years, respectively, regardless of PI use, concurrent $\mathrm{HCV}$, or previous ART (Fig. 4).

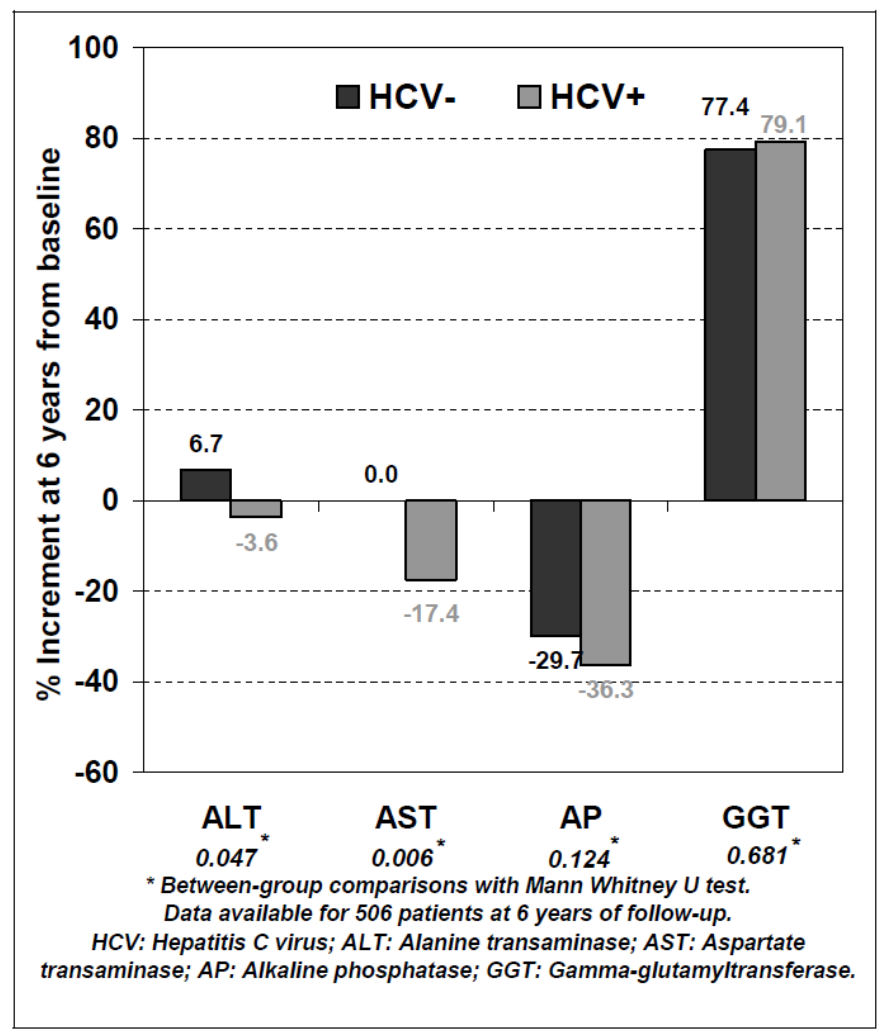

Fig. (4). Percentage increase in hepatic variables $v s \mathrm{HCV}+$ (all patients)

Alkaline phosphatase (AP) decreased in the overall population at 6 and 9 years $(-32.4 \%$ and $-41.3 \%$; both, $\mathrm{p}<0.001)$. AP levels were higher in $\mathrm{HCV}+$ patients at baseline $(\mathrm{p}<0.001)$ and showed a greater decrease in this patient group at follow-up $(-0.87 \mu \mathrm{kat} / \mathrm{L}[-36.3 \%] v s-0.63 \mu \mathrm{kat} / \mathrm{L}[-29.7 \%] ; \mathrm{p}=0.030)$ (Fig. 4). In addition, a larger AP decrease was seen in naïve patients at 6 and 9 years: $-44.3 \%$ vs $-28.3 \%$ $(\mathrm{p}=0.031)$ and $-45.4 \% v s-34.0 \%(\mathrm{p}=0.036)$, respectively (Fig. 2$)$. The AP decrease was smaller, although still statistically significant, in patients receiving tenofovir in their ART regimens.

The Fib-4 biochemical test was carried out to indirectly assess liver fibrosis. In HCV+ patients, Fib-4 score did not change significantly after $6(-1.72)$ or 9 years $(-1.48)$, but it was significantly decreased in naïve patients compared to pretreated patients at these time points: $-29.9 \% v s+14.7 \%(\mathrm{p}<0.001)$ and $22.8 \%$ vs $+25.1 \%(\mathrm{p}=0.003)$, respectively.

In a subgroup of 89 patients, liver fibrosis was determined by FibroScan and/or biopsy; 51 patients (57.3\%) presented at least moderate fibrosis ( $\geq 2$ by biopsy or $\geq 7$ by FibroScan). There were no differences in the ALT, AST, or GGT changes occurring in patients with or without fibrosis, but a greater decrease in AP was found at 6 years in patients with fibrosis $(-47.7 \%$ vs $-9.9 \%, \mathrm{p}=0.009)$. 


\section{DISCUSSION}

After a median period of 9 years, $25.2 \%$ of patients initiating a nevirapine-containing regimen between 1998 and 2003, continued taking nevirapine. As it was not an objective of the study, we do not know the percentage of cases discontinuing the drug for reasons such as virologic failure, toxicity, lost to follow-up, or death. It has been well recognized that a proportion of patients stop nevirapine during the first months of therapy due to adverse effects, and that most patients tolerating nevirapine beyond this period of time will continue to receive it for the long term. It is difficult to compare our data with data from other studies with nevirapine or other drugs as there are no data with such long term follow up. A couple of studies $[9,10]$ have shown that lopinavir/ritonavir and efavirenz are effective and well tolerated after several years of follow-up. But these were prospective clinical trials in which patients are generally selected and closely follow up, making these populations different from the real life. As commented below, in the Eurosida cohort, after a median follow up time of 2.6 years, comparable discontinuation rates of about $50 \%$ were observed amongst nevirapine, efavirenz or lopinavir/ritonavir. Anyway, our data show that after a long period of time, a considerable proportion of patients initiating nevirapine will maintain the drug. Thus, it is clinically relevant to know the long term benefits and limitations of such therapy.

In this long-term study, NVP-containing regimens were associated with an improvement in variables related to general health status, such as hemoglobin and albumin concentration and platelet counts, as well as increased CD4 counts, preserved liver function, and a favorable lipid profile. Our data are consistent with those of previous cohort studies assessing NVP regimens administered for a median of 6 $(n=229)$ and $3.5(n=613)$ years [11, 12]. The long-term safety of NVP with regard to the lipid profile and liver function in cohorts with a high prevalence (30\%-60\%) of HCV+ patients, was also reported in these articles. The large EUROSIDA study recently evaluated the outcomes of patients receiving regimens containing NVP, efavirenz, or lopinavir/ritonavir, and found that NVP durability was comparable to that of the other two regimens in routine clinical practice across Europe in patients who initially tolerated and virologically responded to therapy [13]. The greater discontinuation rate of NVP due to virologic failure was counterbalanced by a lower discontinuation rate due to toxicities or patient/physician choice [13].

In the present study, a continuous increase in absolute CD4 count was observed, with a greater increase in naïve and HCV- patients, as was expected. These findings are in keeping with those of other long-term studies including a smaller number of patients who received lopinavir/r- and efavirenzcontaining regimens $[6,7]$.

NVP use in $\mathrm{HCV}+$ patients is controversial because liver toxicity is one of the main adverse effects occurring in patients receiving this drug [5]. Moreover, liver toxicity associated with the use of ARV drugs is usually somewhat higher in patients coinfected with HCV [4]. Most cases of NVP-associated hepatotoxicity appear during the first 2 to 3 months of therapy and manifest as a hypersensitivity reaction [14-17]. This clinical syndrome seems to be more frequent in patients with certain genetic markers [14], and is mainly driven by the presence of high CD4 counts $(>400$ cells $/ \mu \mathrm{L}$ in men and $>250$ cells $/ \mu \mathrm{L}$ in women), mostly in patients with detectable viral load [17-19]. When NVP is prescribed taking into consideration these CD4 cut-offs, as was done in the ARTEN study, the incidence of rash and liver toxicity is lower than has been previously described [20]. The published data are discordant regarding liver fibrosis in HIV/HCV+ patients receiving NVP or PIs [21, 22].Whereas Macías et al. [21] suggested that NVP regimens may be associated with faster progression of liver fibrosis in HIV/HCV patients when compared to PI regimens, Berenguer et al. [22] reported the opposite; that is, a reduction in fibrosis progression associated with NVP use compared to PIs and efavirenz. Of note, a recent study has suggested that in patients coinfected with $\mathrm{HIV} / \mathrm{HCV}$, interferon/ribavirin treatment is associated with a better response in those that have NVP in their ARV regimen [23].

Our data show that the use of NVP in patients with HIV/HCV infection is relatively safe and provides long-term benefits when examining parameters negatively influenced by liver disease, such as hemoglobin, albumin and platelets, even in patients coinfected with HCV (40\% of our cohort), and a percentage of them with abnormal transaminases at baseline. Moreover, despite this scenario, no 
worsening of transaminases occurred and there was a significantly more favorable change in ALT and AST in $\mathrm{HCV}+$ patients and naïve patients, likely related to the beneficial effect of controlling HIV replication in HCV infection. The finding of a greater decrease in the Fib-4 score in naïve patients coincides with this observation. Concomitant use of a PI did not change the evolution of liver enzymes.

It is well recognized that NVP is associated with an increase in GGT, and this was seen in our patients. As to AP, the notable decrease, mainly observed in naïve patients, $\mathrm{HCV}+$ patients, and the subgroup with liver fibrosis, probably reflects an improvement in liver function in keeping with the transaminase results. Interestingly, patients receiving tenofovir had a significantly lower decrease in AP compared to patients receiving other drugs, suggesting that the known effect of tenofovir on bone AP increase counterbalanced the rise in total AP [24].

NVP is recognized as one of the most lipid-friendly ART drugs [3,4,25-27], and it has been associated with better lipid profiles than other non nucleoside reverse transcriptase inhibitors, such as efavirenz. It also compares favorably with atazanavir, which has the best lipid profile among boosted Pis [22-23]. NVP is the ARV compound associated with the highest HDL-c concentrations, with increases reaching up to $40 \%$ in previous studies $[25,26]$. Our data further support these results, as is seen in the TG decrease and the percentage of patients with HDL-c and LDL-c levels below the cut-off recommended by the NCEP to initiate lipidlowering therapy. As was expected, lipid changes were more favorable in the pretreated patients, most of whom had received previous regimens with a worse lipid profile [26].

This study has some limitations inherent to its retrospective design. One is that fibrosis evaluation by biopsy or FibroScan was only available in a subgroup of patients, limiting the information about the longterm effect of NVP regimens on liver. The same was true for HDL-c and LDL-c levels, which were not routinely determined several years ago.

It can be argued that the study does not provide information on the proportion of patients initiating a NVP regimen who had to discontinue the drug. In fact, we recognize that our patient population is biased because patients discontinuing nevirapine have been excluded. It is well known that a proportion of about 13-20\% of patients initiating a nevirapine-containing regimen will stop the drug because of toxicities/virologic failure in the first year of follow-up, and this has been described in several papers [12, 20]. However, our aim was to focus on the benefits obtained in several relevant clinical aspects occurring in patients initially tolerating NVP and maintaining the regimen in the long term. We believe our study offers valuable long-term information about NVP-containing regimens, in routine clinical practice.

Few studies evaluating ART outcomes after such a long follow-up have been published to date. Our data reinforce the idea that nevirapine-containing regimens provide continuous benefits with regard to viral suppression and immune response, as well as good long-term tolerability, even in HCV-coinfected patients.

\section{ACKNOWLEDGEMENTS}

We thank Celine L Cavallo for her assistance in the correction, editing and proofreading of the manuscript.

\section{CONFLICT OF INTEREST}

The author confirm that this article content has no conflicts on interest. 


\section{DISCLOSURE STATEMENT}

D. Podzamczer, M. Leal, JC López Bernaldo de Quiros, J. Mallolas, E. Ribera, I. Santos and J. Sanz, have received research grants and/or honoraria for advisories and/or conferences from Boehringer Ingelheim, GSK, Viiv, Pfizer, BMS, Abbott, Gilead, Janssen and Merck.

P. Barrufet, MA Cárdenes, L. Force and J López-Aldeguer have received research grants and/or honoraria for advisories and/or conferences from Boehringer Ingelheim, ViiV, BMS, Abbott, Gilead, Janssen and Merck.

JL Gómez-Sirvent has received honoraria for advisories and/or conferences from Boehringer Ingelheim, GSK, Viiv, Pfizer, BMS, Abbott, Gilead, Janssen, Merck and Roche Farma.

S. Moreno has received honoraria for advisories and/or conferences from Abbott, Boehringer\&Ingelheim, Bristol- Myers Squibb, Gilead, Glaxo Smith Kline, Janssen Cilag, Merck Sharp\&Dohme, Pfizer, Roche, Schering Plough, and ViiV Healthcare.

JM Tiraboschi, J. Curto, E. Casas, A. Castro, S. Echevarría, T. Puig, C. Villalonga, JA GarcíaHenarejos have not conflict of interest.

Celine L Carvallo (http://www.celinecavallo.com/) has assisted in the correction, editing and proofreading of the manuscript.

This work was partially financed by Boehringer Ingelheim.

\section{REFERENCES}

[1] European AID Clinical Society. Guidelines: Clinical Management and Treatment of HIV-infected Adults in Europe. April 2011. Version 5-4. Available at: http://europeanaidsclinicalsociety.org/ images/stories/EACS-Pdf/eacs-euroguidelines_fullversion.pdf. Accessed August 1, 2011.

[2] Panel on Antiretroviral Guidelines for Adults and Adolescents. Guidelines for the use of antiretroviral agents in HIV-1-infected adults and adolescents. Department of Health and Human Services. October 14, 2011; 1-174. Available at: http://www.aidsinfo.nih. gov/ContentFiles/AdultandAdolescentGL.pdf. Accessed November 2011.

[3] Martinez E, Conget I, Lozano L, CasamitjanaR, Gatell JM. Reversion of metabolic abnormalities after switching from HIV-1 protease inhibitors to nevirapine. AIDS 1999; 13: 805-10.

[4] Van Leth F, Phanuphak P, Stroes E, et al. Nevirapine and efavirenz elicit different changes in lipid profiles in antiretroviral-therapynaive patients infected with HIV-1. PLoS Med 2004; 1: e19.

[5] Torti C, Costarelli S, De Silvestri A, et al. Analysis of severe hepatic events associated with nevirapinecontaining regimens: CD4+ T-cell count and gender in hepatitis $\mathrm{C}$ seropositive and seronegative patients. Drug Saf 2007; 30: 1161-9.

[6] Cassetti I, Madruga JV, Suleiman JM, et al. The safety and efficacy of tenofovir DF in combination with lamivudine and efavirenz through 6 years in antiretroviral-naïve HIV-1-infected patients. HIV Clin Trials 2007; 8: 164-72.

[7] Murphy RL, da Silva BA, Hicks CB, et al. Seven-year efficacy of a lopinavir/ritonavir-based regimen in antiretroviral-naïve HIV-1- infected patients. HIV Clin Trials 2008; 9: 1-10.

[8] Sterling RK, Lissen E, Clumeck N, et al. Development of a simple noninvasive index to predict significant fibrosis in patients with HIV/HCV coinfection. Hepatology 2006; 43: 1317-25.

[9] Cassetti I, Madruga JVR, A.H. Suleiman JM, et al. The safety and efficacy of tenofovir DF in combination with lamivudine and efavirenz through 6 years in antiretroviral-naïve HIV-1-infected patients. HIV Clin Trials 8(3): 164-72.

[10] Murphy RL, da Silva BA, Hicks CB, et al. Seven-year efficacy of a lopinavir/ritonavir-based regimen in antiretroviral-naïve HIV-1- infected patients. HIV Clin Trials 2008; 9: 1-10.

[11] Bonjoch A, Paredes R, Domingo P, et al. Long-term safety and efficacy of nevirapine-based approaches in HIV type 1-infected patients. AIDS Res Hum Retroviruses 2006; 22: 321-9.

[12] Rodríguez-Arrondo F, Aguirrebengoa K, Portu J, et al. Long-term effectiveness and safety outcomes in HIV-1-infected patients after a median time of 6 years on nevirapine. Curr HIV Res 2009; 7: 526-32. 
[13] Reekie J, Reiss P, Ledergerber B, et al. A comparison of the longterm durability of nevirapine, efavirenz and lopinavir in routine clinical practice in Europe: a EuroSIDA study. HIV Med 2011; 12: $259-68$.

[14] Van Leth F, Phanuphak P, Ruxrungtham K, et al. Comparison of first-line antiretroviral therapy with regimens including nevirapine, efavirenz, or both drugs, plus stavudine and lamivudine: a randomised open-label trial, the 2NN Study. Lancet 2004, 363: 1253-63.

[15] Podzamczer D, Olmo M, Sanz J, et al. Safety of Switching Nevirapine Twice Daily to Nevirapine Once Daily in Virologically Suppressed Patients. J Acquir Immune Defic Syndr 2009; 50: 390- 6.

[16] Yuan J, Guo S, Hall D, et al. Toxicogenomics of nevirapineassociated cutaneous and hepatic adverse events among populations of African, Asian, and European descent. AIDS 2011; 25: 1271-80.

[17] Mocroft A, Staszewski S, Weber R, et al. Risk of discontinuation of nevirapine due to toxicities in antiretroviral-naive and - experienced HIV-infected patients with high and low CD4+ T-cell counts. Antivir Ther 2007; 12: 325-33.

[18] E De Lazzari, A León, JA Arnaiz, et al. Hepatotoxicity of nevirapine in virologically suppressed patients according to gender and CD4 count. HIV Med 2008; 9: 221-6.

[19] Kesselring AM, Wit FW, Sabin CA, et al. Risk factors for treatment-limiting toxicities in patients starting nevirapinecontaining antiretroviral therapy. AIDS 2009; 23(13): 1689-99.

[20] Soriano V, Arasteh K, Migrone, et al. Nevirapine versus atazanavir/ritonavir, each combined with tenofovir disoproxil fumarate/etricitabine, in antiretroviral-naïve HIV-1 patients: the ARTEN trial. Antiviral Therapy 2011:16 (3): 339-48.

[21] Macías J, Castellano V, Merchante N, et al. Effect of antiretroviral drugs on liver fibrosis in HIVinfected patients with chronic hepatitis C: harmful impact of nevirapine. AIDS 2004; 18: 767-74.

[22] Berenguer J, Bellón JM, Miralles P, et al. Association between exposure to nevirapine and reduced liver fibrosis progression in patients with HIV and hepatitis C virus coinfection. Clin Infect Dis 2008; 46: 137-43.

[23] Mira JA, López-Cortes LF, Vispo, et al. Concomitant nevirapine therapy is associated with higher efficacy of pegylated interferon plus ribavirin among HIV/hepatitis $\mathrm{C}$ virus-coinfected patients. XVIII International AIDS Conference. Vienna (Austria) July 2010.

[24] Fux CA, Rauch A, Simcock M, et al. Tenofovir use is associated with an increase in serum alkaline phosphatase in the Swiss HIV Cohort Study. Antivir Ther 2008; 13: 1077-82.

[25] Fisac C, Virgili N, Ferrer E, et al. A comparison of the effects of nevirapine and nelfinavir on metabolism and body habitus in antiretroviral-naive human immunodeficiency virus-infected patients: a randomized controlled study. J Clin Endocrinol Metabol 2003; 88: 5186-92.

[26] Fisac C, Fumero E, Crespo M, et al. Metabolic benefits 24 months after replacing a protease inhibitor with abacavir, efavirenz or nevirapine. AIDS 2005; 19: 917-25.

[27] Podzamczer D, Andrade-Villanueva J, Clotet B, et al. Lipid profiles for nevirapine vs atazanavir/ritonavir, both combined with tenofovir disoproxil fumarate and emtricitabine over 48 weeks, in treatment-naïve HIV-1-infected patients (the ARTEN study). HIV Med 2011; 12: 374-82. 